\title{
Extracorporeal Wound Simulation as a Foundation for Tissue Repair and Regeneration Therapies
}

\section{Hadjipanayi E*, Bekeran S and Moog P}

Experimental Plastic Surgery, Clinic for Plastic and Hand Surgery, Klinikum rechts der Isar, Technische Universität München, Germany

*Corresponding author: Hadjipanayi E, Experimental Plastic Surgery, Clinic for Plastic and Hand Surgery Klinikum rechts der Isar, Technische Universität München, Ismaninger Str. 22, D-81675 Munich, Germany, Tel: +49-(0)89-4140-7338; Fax: +49-(0)89-4140-7336; Email: e.hadjipanayi@gmail.com

\section{Conceptual Paper}

Volume 2 Issue 1

Received Date: April 23, 2018

Published Date: May 21, 2018

DOI: $10.23880 /$ ijtps-16000116

\section{Abstract}

The idea of extracorporeally simulating a wound microenvironment ex vivo in order to obtain growth factor proteins that can then be delivered in vivo to aid wound healing is based on the premise that wound repair operates on a set of complex, yet defined physiological rules. By recapitulating this language, in the form of deliverable growth factor signaling packages, it is possible to effectively influence specific phases of the wound healing program, on demand. The ability to distinctly capture growth factors that are released from cells upon coagulation (coagulation-induced signaling phase) and growth factors that are produced by cells under hypoxia (hypoxia-induced signaling phase) is critical, and represents the most basic segregation of the multi-factorial wound healing cascade. Hypoxia-induced blood-derived secretomes, such as Hypoxia Preconditioned Plasma (HPP) and Hypoxia Preconditioned Serum (HPS), constitute such phase-specific growth factor signaling mixtures that can be obtained through Extracorporeal Wound Simulation (EWS). Novel compositions comprising adjustable concentrations of these secretomes can be added to suitable carriers, and be topically applied or injected into tissue in order to provide angiogenic support and accelerate wound healing and tissue repair. Here we present the research that has laid the foundation for this concept, and discuss how EWS can provide a new platform for developing regenerative therapies.

Keywords: Hypoxia; Blood; Plasma; Preconditioning; Growth Factors; Angiogenesis; Wound 


\section{International Journal of Transplantation \& Plastic Surgery}

Abbreviations: EWS: Extracorporeal Wound Simulation; WBC: white blood cell, PBC: Peripheral Blood Cell; VEGF: Vascular Endothelial Growth Factor; MMPs: Matrix Metalloproteinases; PF-4: Platelet Factor 4; TSP-1: Thrombospondin 1; FGF: Fibroblast Growth Factor; IL-8: Interleukin-8, HPS: Hypoxia Preconditioned Serum; HPP: Hypoxia Preconditioned Serum; PRFM: Platelet-Rich Fibrin Matrix; PBCs: Peripheral Blood Cells; PBMCs: peripheral blood mononuclear cells; PBMNCs: Peripheral Blood Mononuclear Cells; BMCs: Bone Morrow Cells; HDFs: Human Dermal Fibroblasts; CBMSCs: Cord Blood Mesenchymal Stem Cells.

\section{Introduction}

For considerable time, blood has been thought of as a suitable source of growth factor proteins that can be used to aid tissue repair and regeneration [1,2]. Among the various blood-based products that have been tested, platelet-enriched products such as platelet-rich plasma (PRP) / PRP gel and platelet-rich fibrin matrix (PRFM), have received by far the greatest interest $[3,4]$. Specifically, platelet activation and release of factors stored in their granules has long been advocated as a useful strategy for obtaining angiogenic wound healing compositions based on platelet concentrates [4]. This approach is built on the idea that concentrating the number of platelets (e.g. through blood centrifugation) concomitantly increases the concentration of plateletderived growth factors following platelet activation. While this may hold true, it should not be dismissed, that in addition to a whole host of pro-angiogenic mediators (e.g. VEGF), certain factors released by platelets (e.g. PF-4, TSP-1) are strongly anti-angiogenic [5]. Such factors, especially when present in excess (i.e. supraphysiological concentrations, such as those found in platelet concentrates), might negatively impact the angiogenic effectiveness of platelet-rich products, by competing with or negating the effect of pro-angiogenic factors. This could indeed explain the limited success of PRP therapies in treating chronic wounds to date [6]. It is then not unreasonable to argue that the logic behind the development of this method has been based on an incomplete understanding of the complexity, and the fine balance, of physiological growth factor signaling cascades. Anticipating a stronger wound healing response by applying a higher platelet concentration is a simplistic model, one that dismisses the fact that increasing total factor concentration leads to higher concentrations of inhibitors, as well as stimulators.
In contrast to blood centrifugation, and mere concentration of cells/growth factors, a novel alternative approach has emerged, namely that of blood preconditioning through Extracorporeal Wound Simulation (EWS). Conditioning peripheral blood cells (PBCs) under the very same conditions encountered within a wound, i.e. physiological temperature and hypoxia, offers a means for optimizing the angiogenic potential of blood-based products through hypoxiainduced changes in $\mathrm{PBC}$ factor expression, without only relying on the release of platelet-derived factors $[2,7]$. Such secretomes could constitute an improved alternative to platelet-rich products, by specifically providing compositions based on PBCs' natural responses to hypoxic stress, i.e. conditions normally encountered within a healing wound. This presents an opportunity for a paradigm shift in the way blood-derived growth factor mixtures are obtained, applied and function.

\section{Coagulation-Induced Vs. Hypoxia-Induced Wound Healing Signaling Phases}

Blood coagulation is necessary directly after injury to prevent excessive bleeding. At this stage, initiation of new blood vessel formation (angiogenesis) would be logically counterproductive, since newly formed vessels are fragile and unstable. Hemostasis and angiogenesis are therefore two tightly controlled processes, which ensures that angiogenesis is only triggered once hemostasis has been completed [8-10]. This is evident during wound healing, where angiogenesis only begins three days after wounding. This temporal regulation of the hemostatic and angiogenic phases appears to be achieved through the spatiotemporally-controlled release of protein growth factors by cells into the wound microenvironment [10].

Following activation of coagulation, the fibrin clot entraps platelets at the site of injury, forming a hemostatic plug, that is gradually replaced by capillaryrich granulation tissue, and eventually collagen, leading to the restoration of the original extracellular matrix architecture [9]. Platelet activation results in the release of a plethora of growth factors stored in their alphagranules [5]. It is well known, that in addition to various pro-angiogenic mediators, certain factors released by platelets (e.g. PF-4, TSP-1, angiostatin, endostatin) inhibit angiogenesis [5]. Such factors certainly play an important role in the aforementioned natural regulation of the hemostatic and angiogenic phases during wound healing. 


\section{International Journal of Transplantation \& Plastic Surgery}

Nonetheless, the presence of both pro- and antiangiogenic factors in platelet releasates suggests that coagulation-induced signaling might primarily play a regulatory, rather than a directly stimulatory role in new vessel formation.

Clot hypoxia potentiates the platelet-derived proangiogenic signaling generated through coagulation [11]. Hypoxia is the primary angiogenic stimulus in physiological as well as pathological processes [12-15]. Cell accumulation within a growing thrombus or in the maturing wound bed exacerbates the already compromised $\mathrm{O}_{2}$ supply, by increasing local aerobic demand. Cell-mediated hypoxic conditioning of the hemostastic microenvironment results in an upregulated expression of a range of pro-angiogenic growth factors, such as VEGF, ANG, IL8, that are secreted by cells. This effect could be further potentiated though the simultaneous hypoxia-induced downregulation in the expression of anti-angiogenic factors, such as TSP1 [16].

In vitro cellular hypoxic preconditioning has been proposed as a promising strategy for generating complex, yet physiological, angiogenic factor protein mixtures which can be delivered to ischaemic tissues (e.g. wounds, ulcers, burns) to aid re-perfusion, repair and regeneration [15]. Among the various cell types that are suitable for hypoxic stress stimulation, PBCs represent an ideal autologous cell type, since their easy harvest and ample availability means that the need for lengthy cell population expansion cycles, required for example when using biopsy-harvested skin fibroblasts, is circumvented. PBCs can therefore be cultured directly after being obtained from the patient. Previous studies have shown that peripheral blood mononuclear cells (PBMCs) respond to stress (e.g. hypoxia/ischaemia, inflammation) by upregulating a wide range of angiogenic growth factors (e.g. VEGF, bFGF, IL-8, MMP-9) [17-19], and have the ability to induce angiogenesis in vitro, as well as improving central and peripheral vascular perfusion in vivo upon transplantation [19-25]. These findings provide strong evidence that peripheral blood preconditioning is a powerful method for upregulating angiogenic growth factor signaling.

\section{Production of Hypoxia Preconditioned Blood-Derived Secretomes through Extracorporeal Wound Simulation}

Conditioned culture media, containing protein growth factors secreted by cells that are cultured in vitro under normoxia or hypoxia, have previously been administered to promote therapeutic angiogenesis $[15,26]$. A range of cells, including peripheral blood mononuclear cells (PBMNCs) [15,17], bone morrow cells (BMCs) [15,27], mesenchymal stem cells $(15 ; 28)$, human dermal fibroblasts (HDFs) [15,29], human adipose-derived stromal cells (hADSCs) $[15,30]$, cord blood mesenchymal stem cells (CBMSCs) $[15,18]$ and human umbilical vein endothelial cells (HUVECs) $[15,31]$ has been tested and found to positively respond to hypoxic stress by upregulating angiogenic factor signaling. However, as mentioned above, peripheral blood cells offer the unique advantage of easy harvest in numbers sufficiently high, so as to overcome the need for prior cell population expansion. Furthermore, autologous blood products are safe and eliminate the risks associated with inflammatory/immune reactions.

The feasibility of utilizing EWS is based on the simple idea that peripheral blood provides a complete cell culture medium, i.e. comprises the key ingredients necessary for creating a cell culture microenvironment: cells and nutrient medium (plasma). We previously described the use of Hypoxia Preconditioned Plasma (HPP), i.e. plasma derived after extracorporeal conditioning of anti-coagulated blood under physiological temperature $\left(37^{\circ} \mathrm{C}\right)$ and physiological hypoxia $(1-10 \%$ $\mathrm{O}_{2}$ ), as a novel medium for tissue regeneration [7] (Figure 1A). Similarly, conditioning blood in a non-anticoagulated state yields Hypoxia Preconditioned Serum (HPS) (Figure 1A). HPP and HPS represent a special form of conditioned culture medium, in that their composition (concentrations and ratios of factors) is stoichiometrically (i.e. precisely) defined by the patient's peripheral blood cell phenotype and number/count, in contrast to conditioned media typically obtained by ex vivo / in vitro reconstituted culture methods. This confers an important advantage when considering the inter-individual variation present in terms of gene expression and growth factor-induced cellular responses, and forms the basis for their utilization as an autologous therapy.

According to this development, two factors evidently play a key role in carrying out the EWS process: temperature and oxygen tension. First, It is well established that cellular metabolic activity correlates with protein synthetic activity [32]. Angiogenic factor expression by cultured PBCs will also depend on the ambient temperature, a direct controller of cellular metabolism [33]. Culturing PBCs under physiological temperature $\left(37^{\circ} \mathrm{C}\right)$ is therefore preferable to the application of room temperature, as this will promote higher growth factor production [7]. 


\section{International Journal of Transplantation \& Plastic Surgery}

A

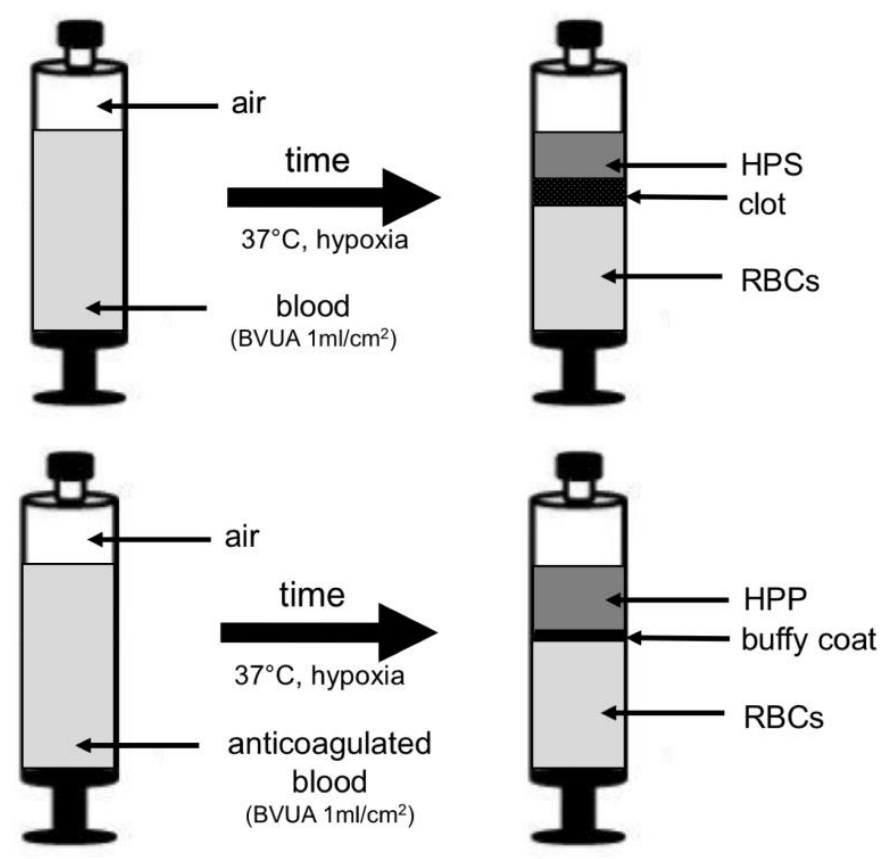

B

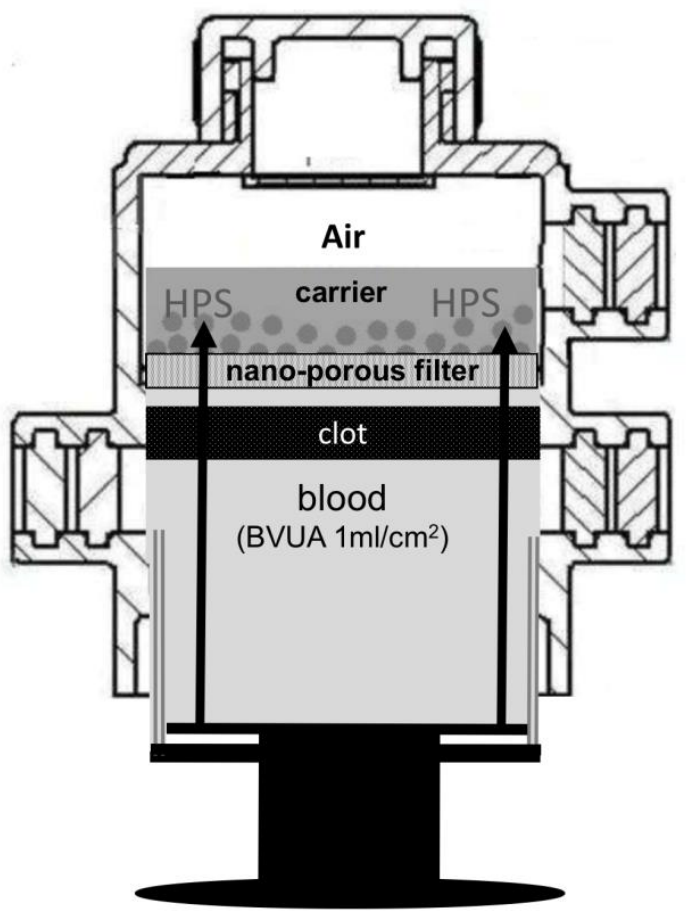

Figure 1: A) Methods for production of Hypoxia Preconditioned Plasma (HPP) and Hypoxia Preconditioned Serum (HPS). BVUA=blood volume per unit (cross-sectional) area of the container. Pericellular hypoxia is generated in situ through cellular $\mathrm{O}_{2}$ consumption under specified BVUA (and PBC seeding density), i.e. no $\mathrm{O}_{2}$ controlled incubator is required. B) EWS device for one-step preparation of sterile carriers loaded with growth factors present in bloodderived secretomes (e.g. HPS) (Device schematic adapted from (2)).

In terms of controlling $\mathrm{O}_{2}$ tension, hypoxic conditioning faces the common problem that generally hinders the application of hypoxia-based therapies, namely the limited availability of $\mathrm{O}_{2}$-controlled incubators/chambers in clinical settings. A promising approach to overcome this limitation is by allowing PBCs to self-regulate their $\mathrm{O}_{2}$ microenvironment, instead of exposing them to an artificial one, i.e. hypoxia produced within an incubator. It has been demonstrated that cell-mediated hypoxia can be achieved in the core of 3D collagen matrix depots, seeded at high density with dermal fibroblasts or vascular smooth muscle cells, by adjusting the total cell number and cell distribution within the depot [29]. A VEGF response was elicited by cells exposed to low levels of $\mathrm{O}_{2}$ $\left(\sim 3 \% \mathrm{O}_{2}\right)$, primarily within the construct core. Also, grafting cord blood mesenchymal stem cells as spheroids in ischaemic hindlimbs of mice was shown to improve therapeutic efficacy due to enhanced cell survival and paracrine activity, effects mediated by hypoxic cell preconditioning within spheroid cultures (in this study, culturing cells as monolayer, where cells were not exposed to hypoxia, abrogated these effects) [18]. These findings therefore indicate that cell-mediated hypoxia is an appropriate alternative to externally-controlled (i.e. incubator) hypoxia. $\mathrm{PBC}$ pericellular $\mathrm{O}_{2}$ tension, being a function of cellular $\mathrm{O}_{2}$ consumption, will predictably dependent on the level of aerobic metabolism (and thus ambient temperature [33]), as well as the population cell number/viability. Both these parameters are directly related to the PBC seeding density, which is initially determined by the ratio of blood volume to cross sectional surface area of the blood container. While lower ratios (e.g. $<0.25 \mathrm{ml} / \mathrm{cm}^{2}$ ) would guarantee uniform cell distribution/spread, and would therefore be more suitable to external (i.e. incubator-controlled) hypoxia, higher ratios (e.g. $0.5-1.5 \mathrm{ml} / \mathrm{cm}^{2}$ ) would produce a higher cell density, therefore aiding the induction of persisting cell-mediated hypoxia. Our findings indicate that a cell seeding density of $\sim 5 \times 10^{6} \mathrm{WBCs} / \mathrm{cm}^{2}$, corresponding to a blood volume per unit area (BVUA) of 


\section{International Journal of Transplantation \& Plastic Surgery}

$1 \mathrm{ml} / \mathrm{cm}^{2}$, is sufficient for maintenance of severe pericellular hypoxia $\left(<1 \% \mathrm{O}_{2}\right)$ over 7 days in cultures of blood obtained from healthy young subjects $[7,10]$.

Devices, such as the one shown in Figure 1B, that can house all steps of the EWS process, including sterile filtration of the blood-derived secretome and one-step loading of growth factors onto a carrier, could enable the rapid preparation of sterile wound dressings and should therefore facilitate the clinical translation of this strategy [2].

\section{Specific Targeting of Wound Healing and Tissue Regeneration Processes}

Any trauma to tissue results in a wound, regardless of the form of insult applied. By definition, a wound represents an interruption in tissue continuity, which can only be repaired through the laying down of a new biomaterial substance. The first biomaterial produced into the wound is fibrin, following activation of the coagulation cascade. Fibrin plays a binary role, acting both as a scaffold for the anchoring, migration and proliferation of cells, as well as a growth factor reservoir $[9,10]$. Indeed, the ability of the fibrin matrix to act as a factor carrier may even extend as far as it playing a key regulatory role, orchestrating the binding and releasing of pro- and anti-angiogenic growth factors, secreted into the wound through coagulation and hypoxia. This would place the fibrin matrix (and fibrinolysis) in the core of the putative biochemical switch mechanism that is responsible for triggering the progression of the hemostatic phase to the angiogenic phase during wound healing [10]. Thus, the ability to deliver a physiological mixture of growth factor proteins within a fibrin-based vehicle would offer the advantage that the factor carrier could concurrently serve as a functional scaffold template upon which tissue repair can materialize.

HPP and HPS differ in that the latter comprises no fibrin(ogen), as this is consumed during blood coagulation. However, their difference extends to their growth factor composition; while HPP comprises exclusively the hypoxia-induced signaling phase (note: this is theoretical, in practice some degree of platelet activation and release of platelet-derived factors does occur during blood incubation), and is thus specifically potent as a pro-angiogenic mixture, HPS represents a more 'complete' secretome, since it provides both the coagulation-induced phase, as well as the hypoxiainduced phase of the wound healing cascade. For example, HPP comprises a significantly greater amount (approx. 5-10 fold higher) of the pro-angiogenic factor VEGF than normal plasma and serum, while containing significantly lower amounts of the angiogenic inhibitors TSP-1 and PF-4 than HPS and PRP (Figure 2). This favorable balance of pro- to anti-angiogenic factors may make HPP an ideal secretome for stimulating angiogenesis on-demand. However, given that both the coagulation-induced phase and the hypoxia-induced phase play an integral role during wound repair, HPS may prove to be more suitable for the treatment of chronic wounds, i.e. wounds that lack the ability to progress to complete healing due to underlying pathology (e.g. diabetic ulcers). In this context, it should not be forgotten that a chronic wound, for it to have a chance of healing, should first be converted to an acute one (e.g. through debridement). This process of 'resetting the clock' makes the necessity for administrating a composition that delivers the complete package of growth factor signals more profound. Ongoing clinical trials by our group appear to support this idea (unpublished data).

In contrast to wound repair following trauma or pathology, the regeneration of (largely) intact tissue that has been altered trough non-traumatic processes, such as aging or photodamaging, as well as controlled dermabrasion (e.g. laser, chemical or mechanical dermabrasion) does not (at least from a macroscopic standpoint) require the initial addition of an external biomaterial scaffold. Yet, it is established that both aging as well as photodamage of skin do result in structural changes in the existing extracellular matrix (ECM) architecture [34]. Any attempt at repairing the ECM must then rely on supporting the innate cellular mechanisms that naturally facilitate tissue repair. Controlled delivery of growth factor signals that promote cell migration towards, and proliferation within the target tissue could be effective by promoting a cellular-driven type of 'in vivo tissue engineering'. To this extent, sufficient angiogenic induction is the primary limiting step, as the survival of newly built tissue ultimately relies on blood-derived nutrient and oxygen support [35]. As postulated above, the utilization of secretomes such as HPP, capable of specifically delivering hypoxia-induced, i.e. angiogenesistargeting growth factors, may prove to be useful for causally overcoming this hurdle.

Spatial growth factor gradients promote chemotactic migration of cells, including endothelial cells towards a target site, and are important for stimulation of directional angiogenesis [36]. To achieve such gradients, growth factors derived through EWS could be loaded onto suitable polymeric carriers that can be topically applied 


\section{International Journal of Transplantation \& Plastic Surgery}

or be locally injected intradermally/subcutaneously through gel or sol-gel vehicles. In addition to using exogenous vehicles, localized delivery could be achieved through the fibrin matrix obtained via the activation of fibrinogenesis in HPP [7]. On the other hand, since HPS is devoid of fibrinogen, exogenous fibrinogen can be added (extra fibrinogen can also be optionally added to HPP, to augment fibrin matrix volume/density if desired). Combining these solutions with thrombin/calcium quickly induces the formation of a fibrin gel-matrix, similar to the one that naturally forms upon blood coagulation. The in vivo-formed fibrin matrix then sequesters the factors at the site of administration, through specific binding (e.g. VEGF) and/or passive trapping, and ensures their controlled release as it occurs within a wound [9]. This also helps to avoid unwanted side-effects such as vascular leakage/edema and ectopic angiogenesis.

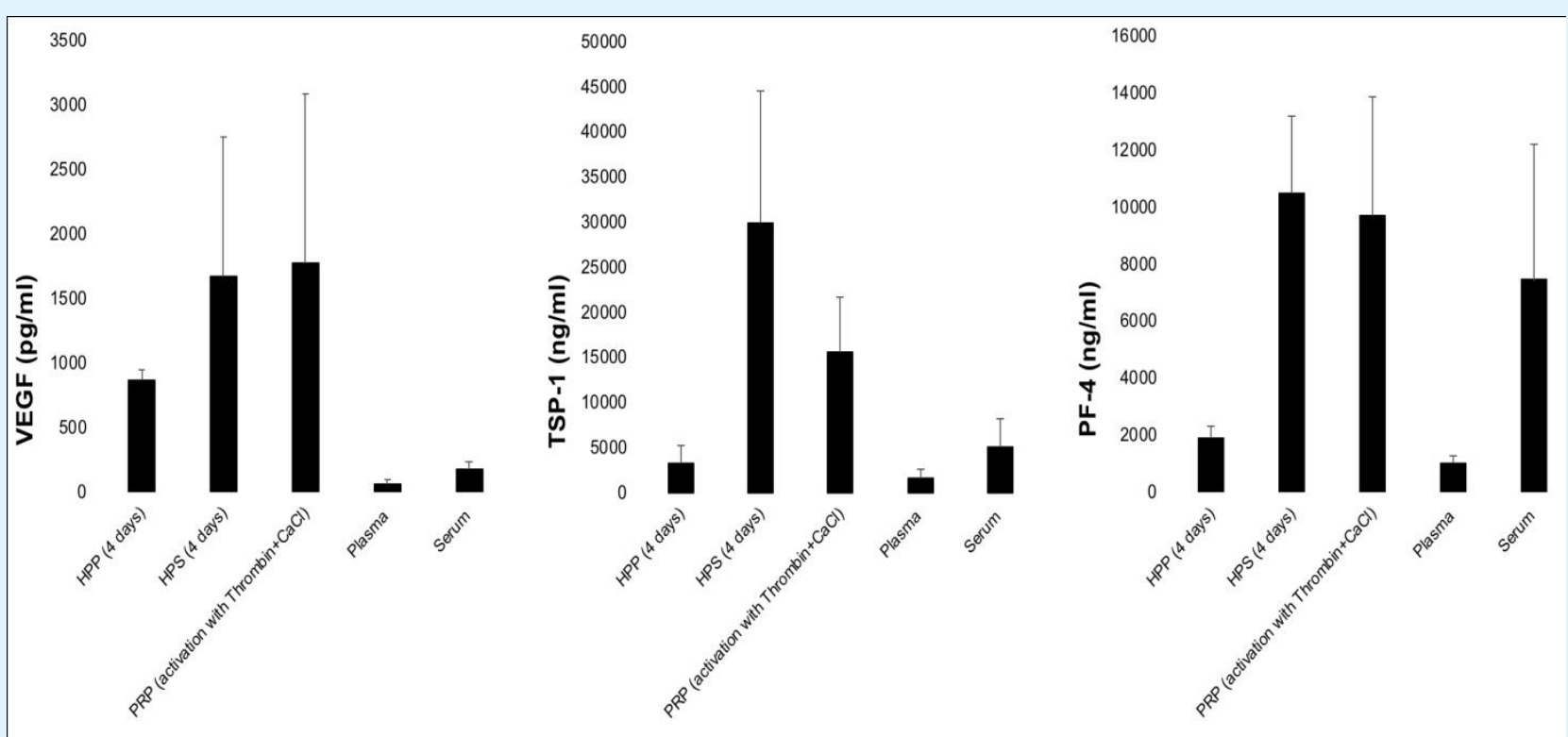

Figure 2: Quantitative analysis of pro-angiogenic factor (VEGF) and anti-angiogenic factor (TSP1, PF-4) protein concentration in different blood-derived secretomes, Hypoxia Preconditioned Plasma (HPP, 4 day incubation), Hypoxia Preconditioned Serum (HPS, 4 day incubation), Platelet-Rich Plasma (PRP), as measured by ELISA. The values were determined from the blood samples of four healthy subjects $(n=4)$. Error bars represent standard deviation of the mean (s.d.).

\section{Novel Compositions with Adjustable} Concentrations of Phase-Specific Growth Factors

As we have already discussed, during wound healing the onset of hypoxia-induced angiogenesis is temporally distinct from the early inflammatory phase, in which platelets are primarily involved in hemostasis (angiogenesis is typically induced 3-4 days after wounding) [9]. While platelets might play a regulatory role, rather than a directly stimulatory role in new vessel formation, their contribution to the initiation, progression and completion of the wound healing process is selfevident. Conclusively, an optimal wound healing composition should comprise both the coagulationinduced, as well as the hypoxia-induced growth factor signaling phases. In nature, these two phases occur sequentially, i.e. the coagulation-mediated phase is induced immediately upon/soon after trauma, through the release of growth factors by activated platelets, followed by the induction of the hypoxia-mediated phase which relies on growth factor production by blood cells and other cells present within the wound. Conditioned culture media, including compositions comprising conditioned blood products (e.g. HPP and HPS), are unable, however, to provide a method for segregating these two phases, as all the factors are released into the same culture medium (plasma or serum). 


\section{International Journal of Transplantation \& Plastic Surgery}

A cumulative mixture is therefore obtained that contains all the required growth factors, but their concentrations and ratios can neither be controlled/adjusted, nor do they correlate with the concentrations/ratios at which these factors are progressively encountered within a natural wound.

The solution to this problem is to develop a method for first separating the two signaling phases, in order to then be able to mix them at defined ratios, in order to obtain tailor-made compositions that can eventually recapitulate the natural sequential expression of the two wound healing phases. Figure 3 describes such a method. After obtaining a peripheral blood sample, the blood is allowed to clot or centrifuged to induce clotting. The serum is then removed completely or partially, without disturbing the clot, and replaced with fresh medium. The fresh medium used for clot incubation can be a suitable cell culture medium (e.g. serum-containing or serum-free culture medium) and/or a solution that is isotonic with blood. Indeed, clinical-grade specialized media for culturing blood cells and for use in ex-vivo tissue processing applications currently do exist (e.g. GIBCO ${ }^{\circledR}$ CTS $^{\mathrm{TM}}$ AIM V ${ }^{\circledR}$ SFM, Therapeutic Grade, Thermo Fisher Scientific Inc.). The clot is incubated in fresh medium at physiological temperature $\left(37^{\circ} \mathrm{C}\right)$ under in situ (cell-mediated) hypoxia (BVUA $\geq 1 \mathrm{ml} / \mathrm{cm}^{2}$ ) for a given time period (4 to 7 days), before collecting the medium. The clot conditioned medium is then mixed with serum at a defined ratio to obtain a novel composition. As is the case for HPP and HPS, the serum and clot conditioned medium can be filtered to remove cells/cellular material, thus providing a cell-free composition.

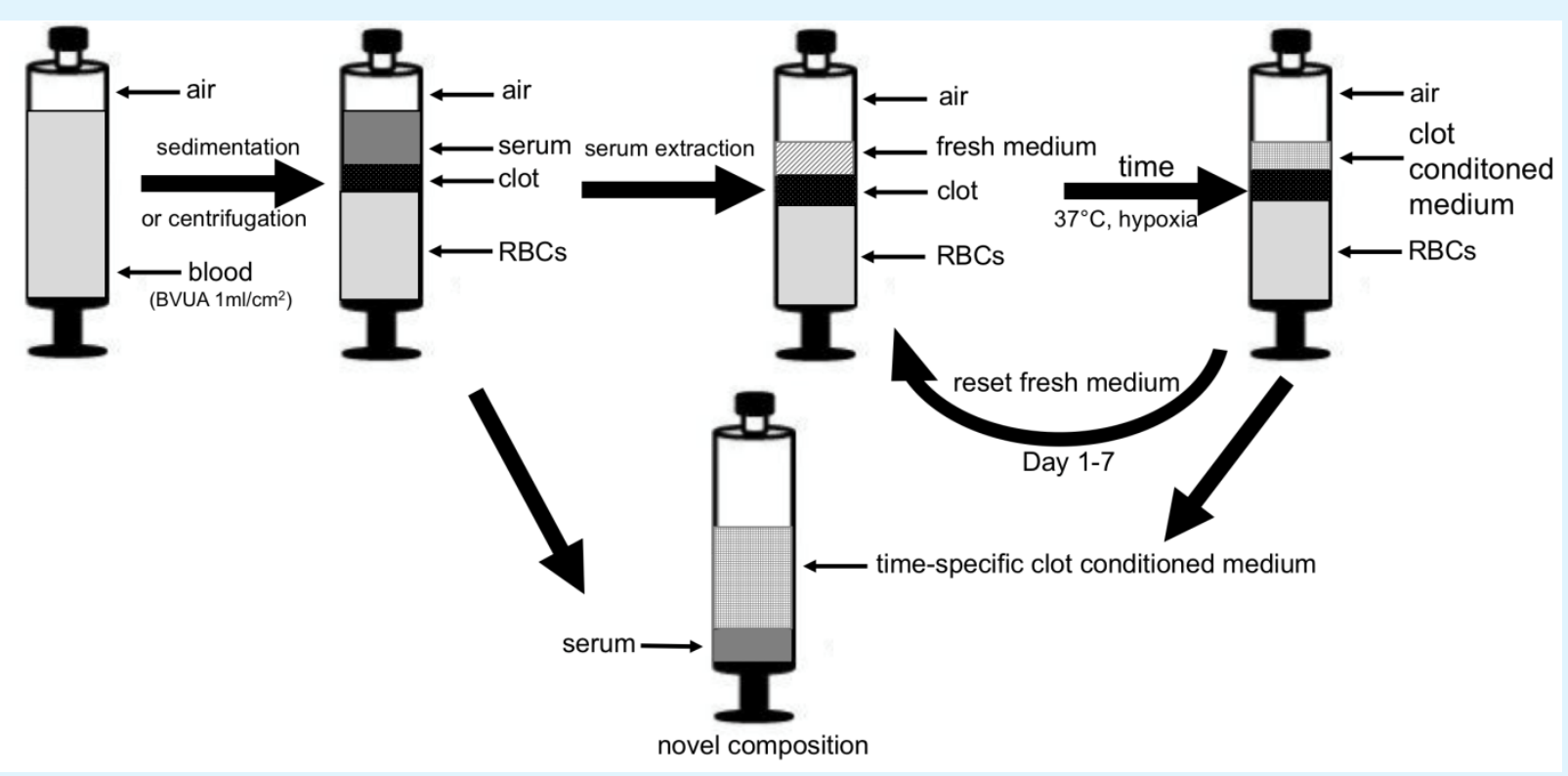

Figure 3: Method for preparing novel compositions comprising the coagulation-induced signaling phase (present in serum) and the hypoxia-induced signaling phase (present in clot conditioned medium) of the wound healing growth factor cascade. Factor concentration can be adjusted by controlling the volume of fresh medium added after serum extraction, and the mixing ratio of the two phases.

This method allows for precise control of the concentration of protein growth factors in clot conditioned medium by controlling the volume of fresh medium added after serum extraction. Preferably, the serum is replaced with fresh medium of a lower volume than the volume of the serum removed, in order to increase the concentration of the factors secreted by blood cells into the conditioned medium above the concentration that would be achieved if the factors were secreted directly into the original serum volume during conditioning. In this way the factors can be concentrated up to 10 -fold, or more. For example, VEGF, which has an average concentration in serum of $60-700 \mathrm{pg} / \mathrm{ml}$ could be concentrated up to $5 \mathrm{ng} / \mathrm{ml}$, and further doubled or tripled through hypoxic conditioning [2,7]. This large increase in factor concentration is significantly higher 


\section{International Journal of Transplantation \& Plastic Surgery}

than the increase that could be ever achieved through blood centrifugation (and platelet enrichment of plasma). The concentration of factors released in the serum can also be adjusted, indirectly, by mixing it with clot conditioned medium. Serum will contain a significantly higher concentration of platelet-derived factors (e.g. PF4) than clot conditioned medium. The preferred mixing volume ratio of clot conditioned medium to serum is such that the concentration of factors in the clot conditioned medium is never diluted, as a result of mixing, below their natural serum concentration. Therefore, the priority is to maintain a high concentration of hypoxia-induced proangiogenic factors, while at the same time also delivering platelet-derived factors at naturally occurring concentrations.

A further idea that derives from this approach is that of temporally-specific growth factor signaling packages. As depicted in figure 3 , clot conditioned medium can be collected at defined time points from the start of incubation, e.g. at 1 to 7 days and replaced by new fresh medium, thus allowing the incubation to continue. Through this process, multiple new compositions can be obtained at defined intervals, that comprise different factor concentrations and ratios, since hypoxia-induced factor expression by PBCs significantly varies over time [2]. These compositions then effectively recapitulate the natural sequential expression of the wound healing cascade. In theory, this would make it possible to selectively intervene at any given time point during wound progression, by delivering compositions that specifically match, from a temporal perspective, the exact stage the wound is currently found (following debridement). Whether this could provide an additional benefit, such as a faster rate of repair, compared to the administration of broader compositions, needs to be examined.

Compositions obtained by these methods could be used to prevent or treat tissue damage by being topically applied onto a wound or injected intradermally/subcutaneously. These modes of administration may also find utility in repairing tissue that has been altered through chronological aging or photoaging. Blood-derived secretomes could also be codelivered with grafts, such as skin and fat grafts, in order to improve their take by increasing early angiogenic support to the graft. This could potentially facilitate the application of grafts into damaged tissue (e.g. irradiated tissue, chronic wounds with compromised blood supply), but could also enable the transplantation/implantation of larger graft volumes. The latter presents an exciting possibility, one that could pave the way for tissue engineering applications.

\section{Conclusion}

The Extracorporeal Wound Simulation (EWS) approach provides significant improvement over currently available systems for preparation of autologous bloodbased compositions, such as platelet-rich products, as it makes it possible to deliver hypoxia-induced (i.e. angiogenesis-targeting) factor mixtures of higher protein concentration and potency, rather than only the factors already present within cells (platelets) at the time of blood collection. By harnessing both the coagulationinduced phase and the hypoxia-induced phase, EWSderived compositions can provide the complete growth factor signalling that normally drives physiological wound healing.

EWS-based therapies can be delivered in the form of wound dressings and injectable preparations, which are entirely cell-free. Importantly, since the protein factors are derived from patient autologous blood, these therapies are safe and patient-specific. By using a personalised therapy, clinicians can better target the wound healing process, as the growth factor requirements for an adequate wound healing response significantly vary between individuals. It will thus have utility both at the bed-side, as an angiogenic therapy in peripheral ischaemic tissue such as wounds, ulcers and burns, as well as pre-, intra- and post-operatively as angiogenic support for central ischaemic tissue, grafts, and engineered implants.

\section{Acknowledgements}

The authors would like to kindly thank Prof. A.F. Schilling and Prof. H.G. Machens for their guidance and support in carrying out the research work presented in this manuscript.

\section{Disclosure Statement}

The device depicted in figure $1 \mathrm{~B}$ of this manuscript is protected by a patent (PCT/EP2013/051910), first filed in Feb. 2012 by Klinikum rechts der Isar, Technische Universität München (Inventors: E.Hadjipanayi, H.G.Machens and A.F.Schilling.). The method described in figure 3 is protected by a patent (Nr. PCT/EP2017/001167), first filed in Dec. 2016 by S. von Isenburg. The work carried out by the authors' group and presented in this manuscript falls under the umbrella of 


\section{International Journal of Transplantation \& Plastic Surgery}

the EmaCure Project (for more info please visit www.emacure.org).

\section{References}

1. Wahl SM, Wong H, McCartney-Francis N (1989) Role of growth factors in inflammation and repair. J Cell Biochem 40(2): 193-199.

2. Hadjipanayi E, Bauer AT, Moog P, Salgin B, Kükrek H, et al. (2013) Cell-free Carrier System for Localised Delivery of Peripheral Blood Cell-Derived Engineered Factor Signaling: Towards Development of a One-Step Device for Autologous Angiogenic Therapy. Journal of Controlled Release 169(1-2): 91-102.

3. Sampson S, Gerhardt M, Mandelbaum B (2008) Platelet rich plasma injection grafts for musculoskeletal injuries: a review. Curr Rev Musculoskelet Med 1(3-4): 165-174.

4. Carter MJ, Flylling C, Parnell LK (2011) Use of platelet rich plasma gel on wound healing: a systematic review and meta-analysis. Eplasty 11: e38.

5. Blair P, Flaumenhaft R (2009) Platelet alphagranules: basic biology and clinical correlates. Blood Rev 23(4): 177-189.

6. Martinez-Zapata MJ, Marti-Carvajal A, Solà I, Expósito JA, Bolíbar I, et al. (2016) Autologous platelet-rich plasma for treating chronic wounds. AR Cochrane Database Syst Rev 25(5): CD006899.

7. Hadjipanayi E, Schlinng AF (2014) Regeneration through autologous hypoxia preconditioned plasma. Organogenesis 10(2): 164-169.

8. Monroe DM, Hoffmann M (2012) The clotting system - a major player in wound healing. Haemophilia 18: 11-16.

9. Reinke JM, Sorg H (2012) Wound repair and regeneration. Eur Surg Res 49(1): 35-43.

10. Hadjipanayi E, Kuhn P, Moog P, Bauer AT, Kuekrek H, et al. (2015) The Fibrin Matrix regulates Angiogenic Responses within the Hemostatic Microenvironment through Biochemical Control. PLoS One 10(8): e0135618.

11. Evans CE, Humphries J, Mattock K, Waltham M, Wadoodi A, et al. (2010) Hypoxia and upregulation of hypoxia-inducible factor 1 alpha\} stimulate venous thrombus recanalization. Arterioscler Thromb Vasc Biol 30(12): 2443-2451.

12. Hickey MM, Simon M (2006) Regulation of angiogenesis by hypoxia and hypoxia-inducible factors. Curr Top Dev Biol 76: 217-257.

13. Rey S, Semenza GL (2010) Hypoxia-inducible factor1-dependent mechanisms of vascularization and vascular remodelling. Cardiovasc Res 86(2): 236-342.

14. Carmeliet P (2000) Mechanisms of angiogenesis and arteriogenesis. Nat Med 6(4): 389-395.

15. Hadjipanayi E, Schilling AT (2013) Hypoxia- based strategies for angiogenic induction. Organogenesis Landes Bioscience 9(4): 261-272.

16. Laderoute KR, Alarcon RM, Brody MD, Calaoagan JM, Chen EY, et al. (2000) Opposing effects of hypoxia on expression of the angiogenic inhibitor thrombospondin 1 and the angiogenic inducer vascular endothelial growth factor. Clin Cancer Res 6(7): 2941-2950.

17. Kubo M, Li TS, Suzuki R, Shirasawa B, Morikage N, et al. (2008) Hypoxic preconditioning increases survival and angiogenic potency of peripheral blood mononuclear cells via oxidative stress resistance. Am J Physiol Heart Circ Physiol 294(2): H590-595.

18. Bhang SH, Li TS, Shin JY, Lee TJ, Kim BS (2012) Transplantation of cord blood mesenchymal stem cells as spheroids enhances vascularization. Tissue Eng Part A 18(19-20): 2138-2147.

19. Lichtenauer $M$, Mildner $M$, Hoetzenecker $K$, Zimmermann M, Podesser BK, et al. (2011) Secretome of apoptotic peripheral blood cells (APOSEC) confers cytoprotection to cardiomyocytes and inhibits tissue remodelling after acute myocardial infarction: a preclinical study. Basic Res Cardiol 106(6): 12831297.

20. Montesano R, Mossaz A, Ryser JE, Orci L, Vassalli P (1984) Leukocyte interleukins induce cultured endothelial cells to produce a highly organized, glycosaminoglycan-rich pericellular matrix. J Cell Biol 99(5): 1706-1715.

21. Bouchentouf M, Paradies P, Forner KA, Cuerquis J, Boivin MN, et al. (2010) Monocyte derivatives 


\section{International Journal of Transplantation \& Plastic Surgery}

promote angiogenesis and myocyte survival in a model of myocardial infarction. Cell Transplant 19(4): 369-386.

22. Kobayashi T, Hamano K, Li TS, Nishida M, Ikenaga S, et al. (2002) Angiogenesis induced by the injection of peripheral leukocytes and platelets. J Surg Res 103(2): 279-286.

23. Kamihata $H$, Matsubara $H$, Nishiue $T$, Fujiyama $S$, Amano K, et al. (2002) Improvement of collateral perfusion and regional function by implantation of peripheral blood mononuclear cells into ischemic hibernating myocardium. Arterioscler Thromb Vasc Biol 22(11): 1804-1810.

24. Iba O, Matsubara H, Nozawa Y, Fujiyama S, Amano K, et al. (2002) Angiogenesis by implantation of peripheral blood mononuclear cells and platelets into ischemic limbs. Circulation 106(15): 2019-2025.

25. Li S, Zhou B, Han ZC. (2006) Therapeutic neovascularization by transplantation of mobilized peripheral blood mononuclear cells for limb ischemia. A comparison between CD34+ and CD34mononuclear cells. Thromb Haemost 95(2): 301-311.

26. Di Santo S, Yang Z, Wyler von Ballmoos M, Voelzmann J, Diehm N, et al. (2009) Novel cell-free strategy for therapeutic angiogenesis: in vitro generated conditioned medium can replace progenitor cell transplantation. PLoS One 4(5): e5643.

27. Kubo M, Li TS, Kurazumi H, Takemoto Y, Ohshima M, et al. (2012) Hypoxic preconditioning enhances angio- genic potential of bone marrow cells with aging- related functional impairment. Circ J 76(4): 986-994.

28. Hu XY, Yu SP, Fraser JL, Lu ZY, Ogle ME, et al. (2008) Transplantation of hypoxia-preconditioned mesenchymal stem cells improves infarcted heart function via enhanced survival of implanted cells and angiogenesis. . J Thorac Cardiovasc Surg 135(4): 799808.

29. Cheema U, Brown RA, Alpa B, MacRobert AJ (2007) Spatially defined oxygen gradients and vascular endothelial growth factor expression in an engineered 3D cell model. Cell Mol Life Sci 65(1): 177-186.

30. Bhang SH, Cho SW, La WG, Lee TJ, Yang HS, et al. (2011) Angiogenesis in ischemic tissue produced by spheroid grafting of human adipose-derived stromal cells. Biomaterials 32(11): 2734-2747.

31. Bhang SH, Lee S, Lee TJ, La WG, Yang HS, et al. (2012) Three-dimensional cell grafting enhances the angiogenic efficacy of human umbilical vein endothelial cells. . Tissue Eng Part A 18(3-4): 310319.

32. Burdon RH (1987) Temperature and animal cell protein synthesis. Symp Soc Exp Biol 41: 113-133.

33. Schulte PM (2015) The effects of temperature on aerobic metabolism: towards a mechanistic understanding of the responses of ectotherms to a changing environment. J Exp Biol 218(12): 18561866.

34. Giacomoni PU, Rein G (2001) Factors of skin aging share common mechanisms. Biogerontology 2(4): 219-229.

35. Nomi M, Miyake H, Sugita Y, Fujisawa M, Soker S (2006) Role of growth factors and endothelial cells in therapeutic angiogenesis and tissue engineering. Curr Stem Cell Res Ther 1(3): 333-343.

36. Barkefors I, Le Jan S, Jakobsson L, Hejll E, Carlson G, et al. (2008) Endothelial cell migration in stable gradients of vascular endothelial growth factor a and fibroblast growth factor 2: Effects on chemotaxis and chemokinesis. J Biol Chem 283(20): 13905-13912. 